\title{
Role of the basolateral amygdala in the reinstatement and extinction of fear responses to a previously extinguished conditioned stimulus
}

\author{
Vincent Laurent and R. Frederick Westbrook ${ }^{1}$ \\ School of Psychology, The University of New South Wales, Sydney, New South Wales 2052, Australia
}

\begin{abstract}
Four experiments used rats to study the role of the basolateral amygdala (BLA) in the reinstatement and extinction of fear responses (freezing) to a previously extinguished conditioned stimulus (CS). In Experiment 1, BLA inactivation before pairing the extinguished CS with the shock unconditioned stimulus (US) or before US-alone exposure impaired the restoration and the reinstatement of fear responses to the extinguished CS. In Experiment 2, BLA inactivation before extinction impaired long-term inhibition of fear responses, but its inactivation before extinction of fear responses restored by CS-US pairing did not impair long-term inhibition. In Experiment 3, BLA inactivation before extinction of fear responses or before the extinction of fear responses reinstated by US-alone exposure impaired long-term inhibition. In Experiment 4, BLA inactivation did not impair long-term inhibition of fear responses reinstated by US-alone exposure if the context where the US-alone exposure occurred had been previously extinguished. These results confirm that the BLA is critical for both learning fear and fear inhibition, but not for relearning this inhibition. The results are consistent with the view that reinstatement is due to the extinguished CS being tested in a dangerous context and are discussed in terms of a contemporary neural model of fear inhibition.
\end{abstract}

Laboratory rats readily learn about novel cues (e.g., those comprising a distinctive context or a discrete stimulus, such as a light) that signal a feared outcome (e.g., brief but aversive foot shock). They exhibit this learning when re-exposed to these cues (the conditioned stimulus, CS) in the absence of the shock unconditioned stimulus (US) in behavioral, autonomic, and endocrine responses indicative of fear in people (Maren 2001; LeDoux 2003). Rats learn to inhibit these fear responses when repeatedly exposed to the CS in the absence of the US. They suppress fear responses across the CS-alone exposures, eventually failing to show any fear responses to the CS. They also retain much of this learned inhibition, showing few fear responses when tested with the CS on the following day. The CS-alone exposures are said to have extinguished fear responses (Myers and Davis 2007). There is considerable evidence that the basolateral complex of the amygdala (BLA) is a critical component of the neural circuits underlying learning to fear context and discrete CSs, as well as learning to inhibit these fears (Maren 2001; Rodrigues et al. 2004; Myers and Davis 2007; Quirk and Mueller 2008). For example, rats subjected to an intra-BLA infusion of drugs, which depress neuronal activity (e.g., the GABAA agonist, muscimol, the $N$-methyl-D-aspartate receptor [NMDAr] antagonist, DL-APV), before exposure to the signaling relation between the CS and the US fail to show fear responses (e.g., freezing, potentiated startle) when subsequently tested drug-free with the CS (Lee and Kim 1998; Wilensky et al. 2006; Laurent and Westbrook 2009b). Rats trained to fear the CS and subjected to a BLA infusion of muscimol or DL-APV before CSalone exposures exhibit a depressed level of fear responses across these exposures. However, these rats show substantial levels of fear responses when subsequently administered a drug-free retention test in comparison to control rats extinguished under vehicle who

\footnotetext{
'Corresponding author.
}

E-mail f.westbrook@unsw.edu.au; fax 61-92-03853044.

Article is online at http://www.learnmem.org/cgi/doi/10.1101//m.1655010. show low levels of fear responses on the retention test (Laurent and Westbrook 2008; Laurent et al. 2008).

Rats relearn to fear an extinguished CS when it again signals an aversive US. They also relearn to inhibit these fear responses when that reconditioned CS is again subjected to extinction. The BLA is critical for relearning to fear an extinguished CS. For example, rats subjected to an intra-BLA infusion of DL-APV before exposure to the signaling relation between the extinguished CS and the US fail to show fear responses when subsequently tested drug-free (Laurent and Westbrook 2009b). This impairment in fear conditioning is not specific to retraining an extinguished CS. For example, rats were conditioned to fear a CS, but not extinguished, and then given a second conditioning trial under a BLA infusion of DL-APV. On the subsequent drug-free test, these rats show levels of fear responses that are similar to those by rats given a single conditioning episode, but are less than those shown by rats administered two conditioning trials (Laurent and Westbrook $2009 b)$. In contrast to this role for the BLA in relearning to fear a CS, relearning to inhibit fear can occur and is even facilitated in the absence of neuronal activity in the BLA. Rats conditioned to fear a context or a discrete CS, extinguished, reconditioned, and then subjected to a BLA infusion of muscimol or the local anesthetic, bupivacaine, before re-extinction show a marked reduction in fear responses across that re-extinction. However, whereas rats extinguished under a BLA infusion of muscimol or bupivacaine subsequently show substantial levels of fear responses on a subsequent drug-free retention test, rats re-extinguished under the drug show just as few fear responses on that test as rats extinguished or re-extinguished under an infusion of vehicle into the BLA (Kim and Richardson 2008; Laurent and Westbrook 2008; Laurent et al. 2008).

There is considerable evidence that the acquisition of fear responses across conditioning is encoded relatively independently of context, whereas their inhibition is encoded against the spatial and temporal cues present across extinction, leading to the restoration of fear responses when the extinguished CS is tested in 
the absence of these cues (Bouton et al. 2006). For example, a CS trained in one context and extinguished in a second context shows "renewal" of fear responses when tested outside the extinction context-either in the training context or in a third context (Harris et al. 2000). Likewise, a CS, which elicits few fear responses when tested shortly after extinction, shows "spontaneous recovery" of fear responses when tested some time after extinction (Leung and Westbrook 2008).

Another manipulation that restores fear responding consists of the interpolation of US-alone exposures between extinction and testing of the extinguished CS. A CS treated in this way shows "reinstatement" of extinguished fear responses (Westbrook et al. 2002). This reinstatement of extinguished fear responses is due to the CS being tested in a context rendered dangerous by US-alone exposures rather than by the US-alone exposures per se (Bouton and Bolles 1979). For example, a CS extinguished and tested in the same context fails to show reinstatement if the US-alone exposures occurred in a different context. However, a CS does show reinstatement if US-alone exposures and testing occur in the same context, while extinction occurs in a different context (Bouton and Bolles 1979). These results have led to the view that conditioning of a context by US-alone exposures returns the subjects to the conditions present when the fear memory was established (conditioning of the background or context across the pairings). This return to the conditions under which acquisition occurred leads to retrieval by the CS of its US associate, rather than of its inhibitory association or the "no US" memory formed across extinction, resulting in its elicitation of fear responses (Bouton et al. 1993). Consistent with this view concerning the role of context conditioning by US-alone exposure, reinstatement fails to occur if US-alone exposures are followed by exposures to the context in the absence of the US. That is, an extinguished CS, which normally shows reinstatement of fear responses when tested in the context where the US-alone exposures had occurred, fails to elicit fear responses if that context had been extinguished before the test (Westbrook et al. 2002).

The present experiments used rats to study the role of the BLA in the reinstatement of fear responses (freezing) by US-alone exposures and in relearning to inhibit these responses across extinction. There were thus two questions of interest.

The first was whether the BLA was critical for the reinstatement of fear responses by US-alone exposures, just as it is critical for the restoration of fear responses produced by pairing an extinguished CS with the US (Laurent and Westbrook 2009b). In Experiment 1, rats were trained to fear a visual CS on day 1, and their fear responses extinguished on day 2. These responses were restored by pairing the CS with the US or they were reinstated by exposure to the US in the absence of the CS on day 3. The pairing or the US-alone exposures occurred under BLA infusion of either muscimol or vehicle. Finally, the levels of fear responses to the CS were assessed on a drug-free retention test on day 4 .

The second question of interest was the role of the BLA in relearning to inhibit fear responses reinstated by US-alone exposure. However, we felt it necessary to first replicate the findings documenting the contrasting role of the BLA in learning to inhibit fear responses across extinction and in relearning this inhibition across a second extinction. These findings have been obtained when fear responses were restored by pairing the extinguished CS with the US and then subjecting that CS to a second extinction (Kim and Richardson 2008; Laurent and Westbrook 2008; Laurent et al. 2008). Therefore, in Experiment 2, rats were trained to fear a visual CS on days 1 and 3, and their fear responses to the CS were extinguished on days 2 and 4 . The second extinction, on day 4 , occurred under BLA infusion of either muscimol or vehicle. Other rats received the same treatment except that the extinction session on day 2 was omitted. Thus, these rats received their initial extinction, on day 4, under BLA infusion of either muscimol or vehicle. Finally, rats received a drug-free retention test on day 5 . The aims of Experiment 3 were to confirm the role of the BLA in learning to inhibit fear responses across extinction and to determine its role in relearning this inhibition across extinction of the fear responses reinstated by US-alone exposure. The design was identical to that used in Experiment 2 except that pairing of the extinguished CS and the US on day 3 was replaced by exposure to the US in the absence of the CS. On day 4 , rats that had received extinction on day 2 underwent a second extinction of the fear responses to the CS reinstated by US-alone exposures. This extinction occurred under BLA infusion of either muscimol or vehicle. On day 4 , rats that had not received extinction on day 2 received their initial extinction under muscimol or vehicle. All rats received a drug-free retention test on day 5 .

In Experiment 3, the rats that had been extinguished to the CS on day 2 and exposed to the US alone on day 3 then received extinction to the CS on day 4 under a BLA infusion of muscimol or saline. This re-extinction of fear responses co-occurred with extinction of the fear responses conditioned to the context by the US-alone exposure on day 3 , thereby confounding re-extinction of fear responses to the CS with extinction of fear responses to context. Experiment 4 examined the role of the BLA in relearning inhibition when both the CS and context had been extinguished in the past. Rats were trained to fear a visual CS on day 1 and their fear responses to this CS were extinguished on day 2. US-alone exposures occurred on days 3 and 5. Half of the rats received context extinction on day 4 and the other half did not. All rats then received a second CS extinction on day 6. This occurred under BLA infusion of muscimol for half of the rats in each group and vehicle for the remainder. Finally, all rats received a drug-free retention test on day 7 .

\section{Results}

\section{Experiment 1: Inactivation of the BLA impairs reconditioning and reinstatement of extinguished fear} As noted previously, the BLA is necessary for both conditioning and reconditioning an extinguished CS (Laurent and Westbrook 2009b). In Experiment 1, we investigated whether the BLA is also necessary for the reinstatement of fear responses to an extinguished CS by US-alone exposure (Fig. 2A, below). Rats were trained to fear a visual CS on day 1 and their fear responses were extinguished on day 2 . Then, the fear responses were restored by subjecting rats to either pairing of the extinguished CS with the US (groups Reconditioning) or to US-alone exposure (groups Reinstatement). Reconditioning and reinstatement was performed under BLA infusion of either muscimol (groups ReconditioningMuscimol and Reinstatement-Muscimol) or vehicle (groups Reconditioning-Vehicle and Reinstatement-Vehicle). The levels of fear were assessed across a drug-free retention test on day 4 .

\section{Histology}

Figure 1A shows the approximate location of injection cannula tips. A total of five rats were excluded because of incorrect placement. This yielded the following group sizes: group ReconditioningVehicle $(n=7)$, group Reconditioning-Muscimol $(n=6)$, group Reinstatement-Vehicle $(n=6)$, and group Reinstatement-Muscimol $(n=7)$.

\section{Behavior}

Levels of freezing to the context and the CS across extinction, reconditioning, reinstatement, and test are shown in Figure 2. The two CS-US pairings on day 1 were successful. All rats exhibited substantial levels of freezing when presented with the CS in 
A

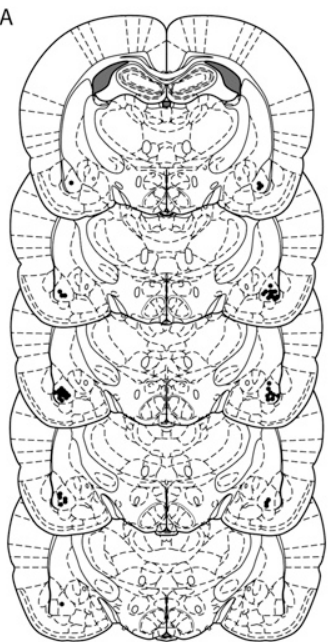

C

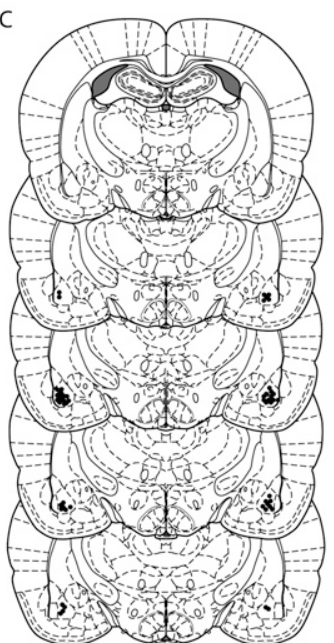

B

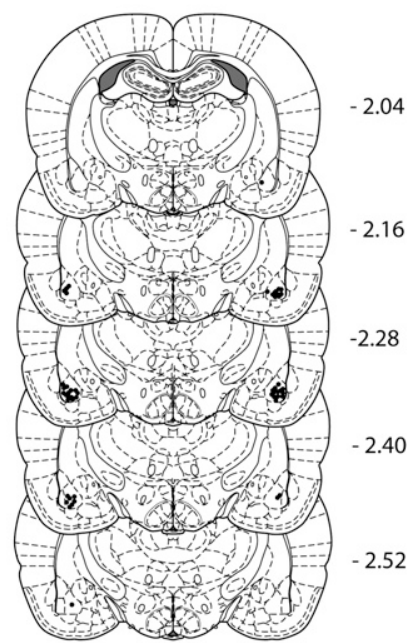

D

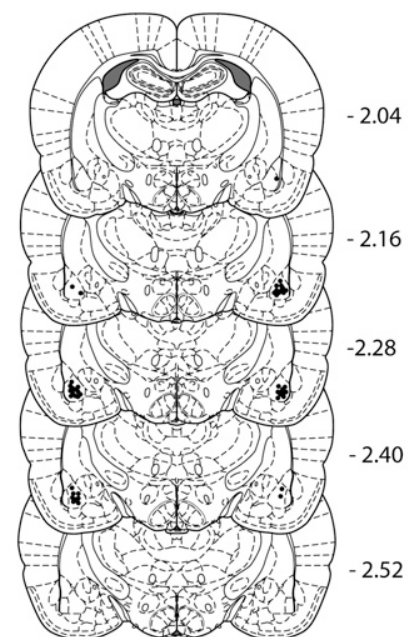

Figure 1. Location of microinjection cannulas within the BLA for all rats included in the final analysis of Experiments $1(A), 2(B), 3(C)$, and $4(D)$. Distances on the atlas templates (adapted from Paxinos and Watson 2007 and reprinted with permission from Elsevier (C2007) are indicated in millimeters from bregma.

extinction on day 2 (Fig. 2B). These levels significantly and gradually declined over the course of the session $\left(F_{(1,22)}=240.5\right.$, $P<0.05)$. Disruption of neuronal activity in the BLA on day 3 depressed fear responses across reconditioning (Fig. 2C, left panel) and US-alone exposures on day 3 (Fig. 2C, right panel). Rats in group Reconditioning-Vehicle froze more than rats in group Reconditioning-Muscimol during the presentation of the CS $\left(F_{(1,11)}=33.8, P<0.05\right)$ and after the administration of the US $\left(F_{(1,11)}=23.7, P<0.05\right)$. Similarly, rats in group ReinstatementVehicle froze more than rats in group Reinstatement-Muscimol after the US-alone exposure $\left(F_{(1,11)}=119.8, P<0.05\right)$.

The retention test data on day 4 (Fig. 2D) showed that neuronal activity in the BLA is necessary for reconditioning and US-alone exposures to restore and reinstate extinguished fear responses. Rats in group Reconditioning-Vehicle froze significantly more to the CS than rats in group Reconditioning-Muscimol $\left(F_{(1,22)}=31.7, P<0.05\right)$. Similarly, rats in group ReinstatementVehicle froze significantly more to the CS than rats in group Reinstatement-Muscimol $\left(F_{(1,22)}=22.0, P<0.05\right)$. There was no statistically significant difference between vehicle-treated rats $\left(F_{(1,22)}=0.8, P>0.05\right)$ or between muscimol-treated rats $\left(F_{(1,22)}=\right.$

$0.0, P>0.05)$. Levels of pre-CS freezing showed that muscimol disrupted the ability of the context to elicit fear responses. Vehicletreated rats exhibited similar levels of fear responses $\left(F_{(1,22)}=5.3\right.$, $P>0.05)$, but significantly higher levels than muscimol-treated rats $\left(F_{(1,22)}=11.9, P<0.05\right)$. There were no statistically significant differences between rats in groups Reconditioning-Muscimol and Reinstatement-Muscimol $\left(F_{(1,22)}=0.0, P>0.05\right)$.

\section{Experiment 2: Inactivation of the BLA impairs extinction of conditioned fear but spares extinction of reconditioned fear}

Previous studies have demonstrated that neuronal activity in the BLA is necessary for learning to inhibit context conditioned fear responses, but not for relearning this inhibition when an extinguished context has been reconditioned and is again extinguished (Laurent and Westbrook 2008; Laurent et al. 2008). In Experiment 2 , we sought to replicate these results using a discrete visual cue CS (flashing light). Rats were bilaterally implanted with cannulas targeting the BLA and randomly assigned to one of the two experimental conditions (Fig. 3A). Rats in groups Re-extinction were trained to fear the CS on day 1 and 3 , and their fear responses were extinguished on days 2 and 4 . The re-extinction session on day 4 was performed under BLA infusion of either muscimol (group Re-extinction-Muscimol) or saline (group Re-extinctionVehicle). Rats in groups Extinction received the same procedure except that the initial extinction session on day 2 was omitted. Finally, the long-term effects of extinction and re-extinction were assessed on a drug-free retention test on day 5 .

\section{Histology}

Figure $1 \mathrm{~B}$ shows the location of injection cannula tips. A total of 12 rats were excluded because of incorrect placement. This yielded the following group sizes: group Extinction-Vehicle $(n=8)$, group Extinction-Muscimol $(n=6)$, group Re-extinction-Vehicle $(n=6)$, and group Re-extinction-Muscimol $(n=7)$.

\section{Behavior}

Levels of freezing to the context and the CS across extinction, reextinction, and test are shown in Figure 3. Conditioning on day 1 was successful. Rats in groups Re-extinction showed substantial levels of freezing when presented with the CS across extinction on day 2 (Fig. 3B) and these levels were higher than those displayed by rats exposed to the context alone (groups Extinction, $F_{(1,23)}=$ $56.7, P<0.05)$. The levels of CS freezing gradually and significantly declined across the session $\left(F_{(1,23)}=45.7, P<0.05\right)$. The CS-US pairing on day 3 restored extinguished fear responses. Rats in group Re-extinction-Vehicle froze just as much as rats in group Extinction-Vehicle when presented with the CS across their respective re-extinction and extinction on day 4 (Fig. 3C, $F_{(1,23)}=$ $0.1, P>0.05$ ). Rats in group Extinction-Vehicle froze significantly less before the onset of the CS than rats in groups Re-extinctionVehicle $\left(F_{(1,23)}=17.3, P<0.05\right)$. This difference is likely due to the greater extinction of any context conditioned fear on day 2 among rats in group Extinction-Vehicle than among rats in group ReExtinction-Vehicle who had been extinguished to the CS on that day. Disrupting neuronal activity in the BLA depressed fear responses. Rats infused with muscimol (groups Extinction-Muscimol and Re-extinction-Muscimol) froze significantly less before $\left(F_{(1,23)}=14.2, P<0.05\right)$ and after the onset of the CS $\left(F_{(1,23)}=138.8\right.$, $P<0.05)$ than rats infused with vehicle. The levels of freezing gradually and significantly decreased over the course of the session $\left(F_{(1,23)}=69.1, P<0.05\right)$.

The retention test data on day 5 (Fig. 3D) confirmed that neuronal activity in the BLA is necessary for learning extinction of fear to a visual CS, but not for relearning extinction to 
A

\begin{tabular}{|lccccc|}
\hline Groups & Day $\mathbf{1}$ & Day $\mathbf{2}$ & Day $\mathbf{3}$ & Day 4 \\
\hline Reconditioning & 2 CS - US & CS & † CS - US & CS \\
\hline Reinstatement & 2 CS - US & CS & †CTX - US & CS \\
\hline
\end{tabular}

个 BLA infusion of vehicle or muscimol $(0.3 \mu \mathrm{g}$ in $0.3 \mu \mathrm{L}$, both sides $)$.

B

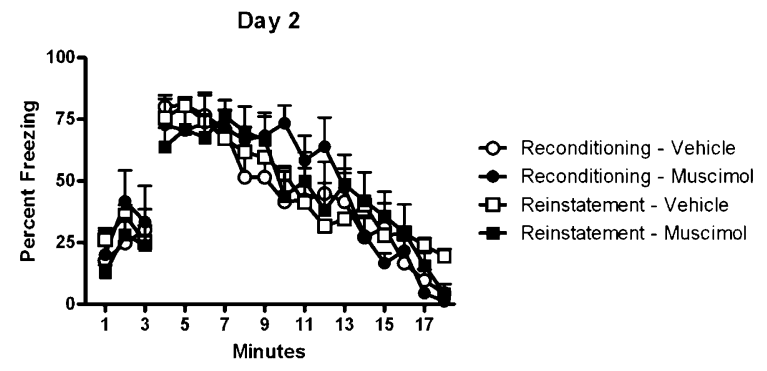

C

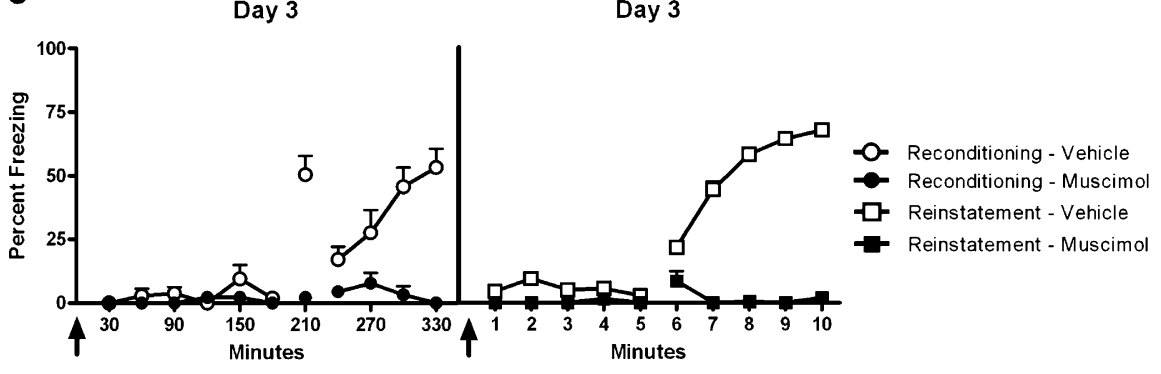

D

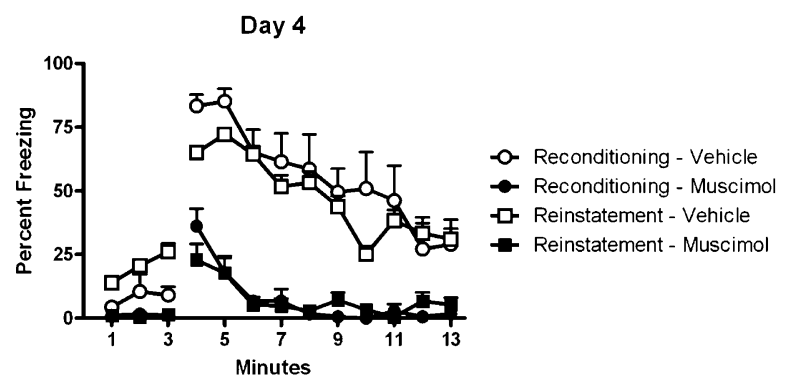

Figure 2. Inactivation of the BLA impairs reconditioning and reinstatement of extinguished fear. $(A)$ Description of the behavioral procedures used in Experiment 1 (CS: flashing light conditioned stimulus; US: footshock unconditioned stimulus; CTX: context). (B) All illustrations show the mean and SEM levels of freezing. All rats lost freezing responses across initial extinction. (C) Rats infused with vehicle in the BLA before reconditioning (left panel; group Reconditioning-Vehicle) or reinstatement (right panel; group Reinstatement-Vehicle) showed more fear responses after the occurrence of the footshock. BLA infusion of muscimol reduced fear responses in both reconditioning (group Reconditioning-Muscimol) and reinstatement (group Reinstatement-Muscimol). $(D)$ The retention test revealed that inactivation of the BLA impaired reconditioning (group Reconditioning-Muscimol) and reinstatement (group Reinstatement-Muscimol) of extinguished fear responses.

a re-conditioned visual CS. Rats in group Extinction-Muscimol froze significantly more to the CS than rats in groups ExtinctionVehicle $\left(F_{(1,23)}=40.9, P<0.05\right)$. In contrast, rats in group Reextinction-Muscimol exhibited just as few fear responses as rats in group Re-extinction-Vehicle $\left(F_{(1,23)}=0.1, P>0.05\right)$. There were no statistically significant differences between the levels of freez- ing by rats in groups Extinction-Vehicle, Re-extinction-Vehicle, and Re-extinctionMuscimol $(F s<2.8)$. Levels of pre-CS freezing did not differ between groups $($ Fs $<3.3)$.

\section{Experiment 3: Inactivation of the BLA impairs learning extinction of reinstated fear} Experiment 2 confirmed that neuronal activity in the BLA is necessary to learn extinction of fear to a discrete CS, but that the fear responses extinguished and restored by reconditioning can be reextinguished in the absence of neuronal activity in the BLA. This suggests that, in the absence of neuronal activity in the BLA, compensatory mechanisms support relearning extinction of fear responses restored by reconditioning (Laurent and Westbrook 2008, 2009a). In Experiment 3, we examined the role of the BLA in relearning extinction of fear responses reinstated by US-alone exposure. The design was the same as in the previous experiment except that pairing of the extinguished CS and the US on day 4 was replaced with exposure to the US alone (Fig. $4 \mathrm{~B})$. Thus, on day 4 , rats were infused in the BLA with either muscimol (groups Extinction-Muscimol and Re-extinctionMuscimol) or saline (groups ExtinctionVehicle and Re-extinction-Vehicle) before extinction of the fear responses reinstated by US-alone exposure on day 3. A control group of rats (group Control Re-extinction) received the same procedure as group Re-extinction except that the second extinction on day 4 was omitted.

\section{Histology}

Figure 1C shows the location of injection cannula tips. A total of nine rats were excluded because of incorrect placements. This yielded the following group sizes: group Extinction-Vehicle $(n=5)$, group Extinction-Muscimol $(n=5)$, group Re-extinction-Vehicle $(n=7)$, group Re-extinction-Muscimol $(n=5)$, and group Control Re-extinction $(n=7)$.

\section{Behavior}

Levels of freezing to the context and the CS across extinction, re-extinction, and test are shown in Figure 4. Conditioning of the visual stimulus on day 1 was successful. Rats in groups Control Re-extinction and Re-extinction showed substantial levels of freezing when presented with the visual CS for extinction on day 2 (Fig. 4B). These levels were higher than those displayed by rats exposed to the context alone (groups Extinction, $\left.F_{(1,24)}=64.5, P<0.05\right)$. Levels of freezing significantly and gradually declined over the course of the session $\left(F_{(1,23)}=34.4, P<\right.$ $0.05)$. The US-alone exposure on day 3 reinstated extinguished 
A

\begin{tabular}{|lccccc|}
\hline Groups & Day $\mathbf{1}$ & Day $\mathbf{2}$ & Day $\mathbf{3}$ & Day 4 & Day $\mathbf{5}$ \\
\hline Extinction & $2 \mathrm{CS}-$ US & CTX & CS - US & 个CS & CS \\
\hline Re-extinction & 2 CS - US & CS & CS - US & †CS & CS \\
\hline
\end{tabular}

BLA infusion of vehicle or muscimol $(0.3 \mu \mathrm{g}$ in $0.3 \mu \mathrm{L}$, both sides).

B

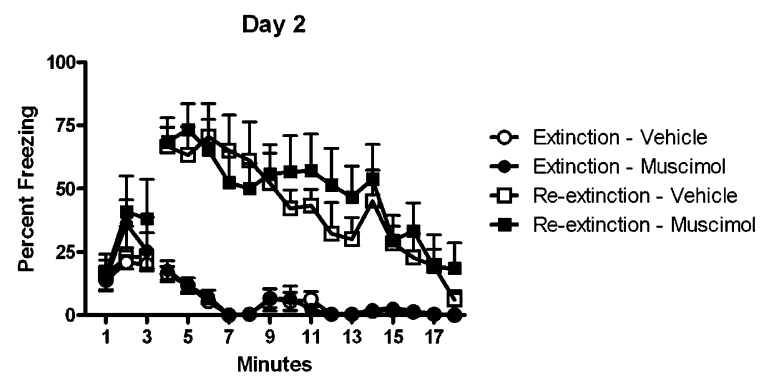

C

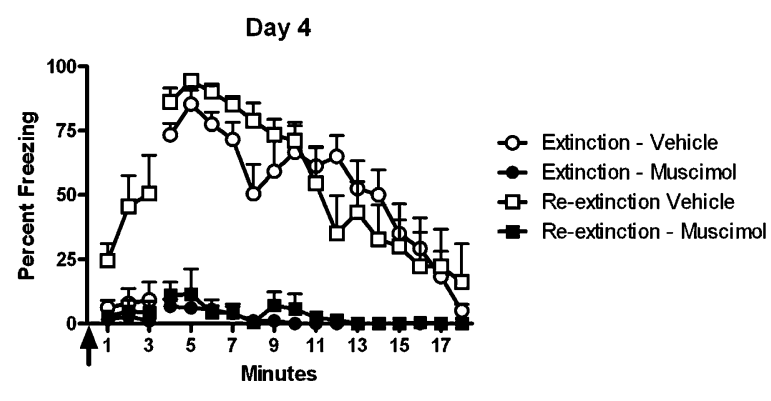

D

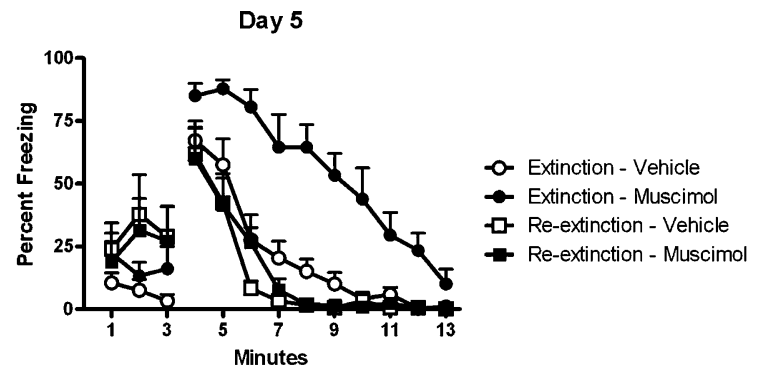

Figure 3. Inactivation of the BLA impairs extinction of conditioned fear, but spares extinction of reconditioned fear. $(A)$ Description of the behavioral procedures used in Experiment 2 (CS: flashing light conditioned stimulus; US: footshock unconditioned stimulus; CTX: context). (B) All illustrations show the mean and SEM levels of freezing. All rats submitted to extinction of the light CS lost freezing responses across initial extinction (group Re-extinction). (C) Rats infused with vehicle before their first (group Extinction-Vehicle) or their second (group Re-extinction-Vehicle) extinction lost freezing responses across the session. BLA infusion of muscimol reduces fear responses in both extinction and re-extinction (groups Extinction-Muscimol and Re-extinction-Muscimol). (D) The retention test showed that inactivation of the BLA impairs learning extinction of conditioned fear (group ExtinctionMuscimol), while sparing learning extinction of reconditioned fear (Re-extinction-Muscimol).

fear responses. Rats in group Re-extinction-Vehicle exhibited substantial and similar levels of freezing to the CS on day 4 as rats in group Extinction-Vehicle that had been extinguished to the context but not to the CS on day $2\left(F_{(1,18)}=0.1, P>0.05\right.$; Fig 4C). BLA infusion of muscimol depressed fear responses. Rats in groups Extinction-Muscimol and Re-extinction-Muscimol froze significantly less before and after the onset of the CS than vehicle- treated rats $\left(F_{(1,18)}=4.6, P<0.05\right.$ and $F_{(1,18)}=72.7, P<0.05$, respectively). The levels of freezing significantly and gradually declined over the course of the session $\left(F_{(1,18)}=26.6, P<0.05\right)$.

The retention test data on day 5 (Fig. 4D) showed that neuronal activity in the BLA is required for learning extinction to both conditioned fear and reinstated fear. Rats in group Extinction-Muscimol froze more to the CS than rats in group Extinction-Vehicle $\left(F_{(1,23)}=13.1, P<\right.$ $0.05)$. Similarly, rats in group Re-extinction-Muscimol froze more to the CS than rats in group Re-extinction-Vehicle $\left(F_{(1,23)}=34.1, P<0.05\right)$. There was no significant difference between vehicletreated rats $\left(F_{(1,23)}=0.5, P>0.05\right)$ or between muscimol-treated rats $\left(F_{(1,23)}=\right.$ $1.3, P>0.05)$. The infusion of muscimol by itself was without effect since rats in group Control Re-extinction showed equivalent levels of CS freezing regardless of whether they were infused in the BLA with either saline or muscimol $\left(F_{(1,23)}=1.1, P>0.05\right)$. Rats that had not received the second extinction on day 4 showed similar levels of CS freezing as rats in groups Extinction-Muscimol and Re-extinction-Muscimol $\left(F_{(1,23)}=3.7\right.$, $P>0.05)$. Levels of pre-CS freezing indicated that rats in group Control Re-extinction froze more before the onset of the CS than all other groups $\left(F_{(1,24)}=6.6\right.$, $P<0.05)$.

\section{Experiment 4: Inactivation of the} BLA spares learning extinction of reinstated fear if the reinstatement context has been extinguished

In Experiment 3, rats were administered US-alone exposures and the reinstated fear responses were extinguished under either a BLA infusion of muscimol or vehicle. The subsequent retention test revealed that rats infused with muscimol across extinction of the reinstated fear responses continued to show fear responses, indicating that extinction had been impaired by inactivation of neuronal activity in the BLA. However, inactivation of the BLA would also have impaired any extinction of the fear conditioned to the context by the US-alone exposures. Hence, rats infused with muscimol across extinction of the reinstated fear responses may have continued to show such responses on tests because the context remained dangerous as a result of the US-alone exposures. As noted previously, the BLA is critical for extinction of context conditioned fear but not for re-extinction of such fear (Laurent et al. 2008). Therefore, Experiment 4 examined the effects of extinguishing fear responses to an extinguished CS in rats that had or had not been previously subjected to context extinction (Fig. 5A). Rats in groups Context Extinction were 
A

\begin{tabular}{|c|c|c|c|c|c|}
\hline Groups & Day 1 & Day 2 & Day 3 & Day 4 & Day 5 \\
\hline Control Re-extinction & $2 \mathrm{CS}-\mathrm{US}$ & CS & CTX - US & $\uparrow$ & CS \\
\hline Extinction & $2 \mathrm{CS}-\mathrm{US}$ & CTX & CTX - US & $\boldsymbol{\varphi} \mathrm{Cs}$ & CS \\
\hline Re-extinction & $2 \mathrm{CS}-\mathrm{US}$ & CS & CTX - US & $\uparrow \mathrm{CS}$ & CS \\
\hline
\end{tabular}

BLA infusion of vehicle or muscimol $(0.3 \mu \mathrm{g}$ in $0.3 \mu \mathrm{L}$, both sides).

B

Day 2

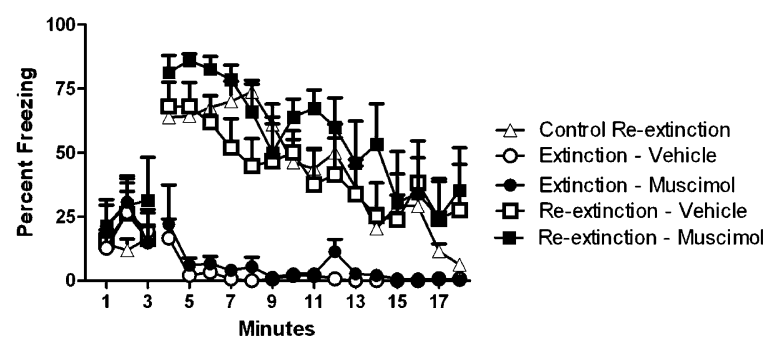

C

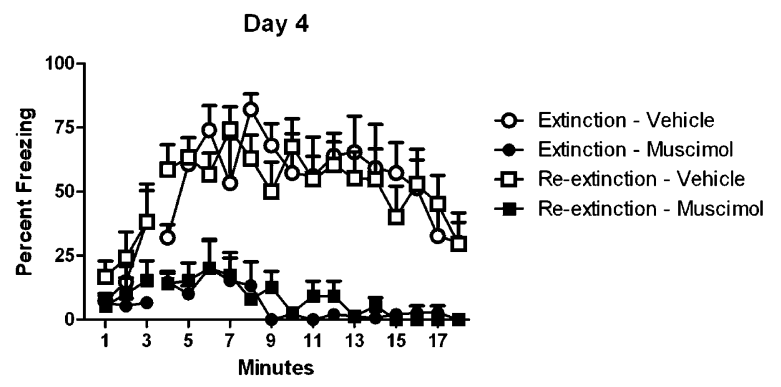

D

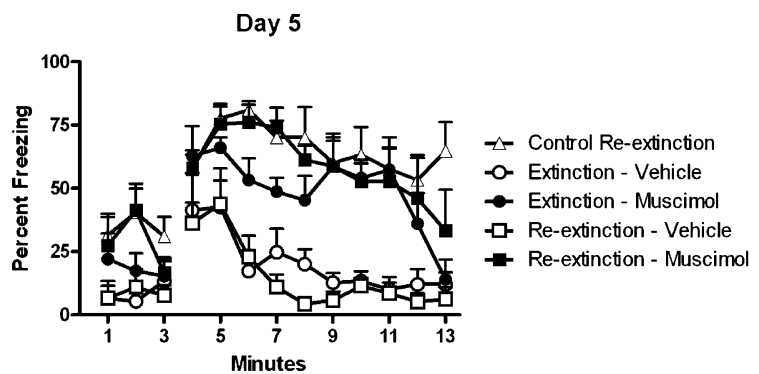

Figure 4. Inactivation of the BLA impairs learning extinction of reinstated fear. $(A)$ Description of the behavioral procedures used in Experiment 3 (CS: flashing light conditioned stimulus; US: footshock unconditioned stimulus; CTX: context). (B) All illustrations show the mean and SEM levels of freezing. All rats submitted to extinction of the light CS lost freezing responses across initial extinction (groups Re-extinction and Control Re-extinction). (C) Rats infused with vehicle before their first (group Extinction-Vehicle) or their second (group Re-extinction-Vehicle) extinction lost freezing responses across the session. BLA infusion of muscimol reduces fear responses in both extinction and re-extinction (groups Extinction-Muscimol and Re-extinction-Muscimol). (D) The retention test showed that BLA inactivation impairs learning extinction of conditioned fear (group Extinction-Muscimol) and reinstated fear (group Re-extinction-Muscimol).

trained to fear a visual CS on day 1 and their fear responses to this CS were extinguished on day 2. Then, they received US-alone exposures on days 3 and 5, and a context-alone exposure on day 4 . Rats in groups No Context Extinction were treated in an identical manner except that the context-alone exposure on day 4 was omitted. All rats received a re-extinction session to the visual CS on day 6 under a BLA infusion of either muscimol (groups No Context Extinction-Muscimol and Context ExtinctionMuscimol) or vehicle (groups No Context Extinction-Vehicle and Context Extinction-Vehicle). Finally, long-term re-extinction to the visual CS was assessed on a drug-free retention test on day 7 .

We hypothesized that muscimoltreated rats will re-extinguish fear responses to both the discrete CS and the dangerous context on day 6 if they had received context-alone exposure on day 4 (group Context Extinction-Muscimol), since we have shown that relearning extinction of context reconditioned fear occurs in the absence of neuronal activity in the BLA (Laurent et al. 2008). Therefore, these rats will exhibit relatively few fear responses on the retention test since the context is no longer dangerous. In contrast, muscimol-treated rats that had not received context-alone exposure on day 4 (group No Context ExtinctionMuscimol) will not show context extinction and, therefore, will exhibit the high levels of freezing to the visual CS at test similar to the results found in Experiment 3.

\section{Histology}

Figure 1D shows the approximate location of injection cannula tips. A total of six rats were excluded because of incorrect placement. This yielded the following group sizes: group No Context Extinction-Vehicle $(n=6)$, group No Context Extinction-Muscimol $(n=7)$, group Context Extinction-Vehicle $(n=6)$, and group Context Extinction-Muscimol $(n=$ 6).

\section{Behavior}

Levels of freezing to the context and the CS across extinction, context-alone exposure, re-extinction, and test are shown in Figure 5. Conditioning on day 1 was successful. All rats showed substantial levels of freezing when presented with the CS for extinction on day 2 (Fig. 5B). These levels gradually and significantly declined across the session $\left(F_{(1,21)}=\right.$ 214.3, $P<0.05)$. The US-alone exposure on day 3 produced substantial levels of context fear when rats in groups Context Extinction were returned to the conditioning chambers on day 4 (Fig. 5C). These levels significantly and gradually declined across this context-alone exposure $\left(F_{(1,10)}=129.0, P<0.05\right)$. The second US-alone exposure on day 5 restored fear responses to the extinguished visual CS. Rats in groups No Context Extinction-Vehicle and Context Extinction-Vehicle showed similar $\left(F_{(1,21)}=0.2, P>\right.$ $0.05)$ and substantial levels of freezing when presented with the visual CS for re-extinction on day 6 (Fig. 5D). Muscimol infused in the BLA depressed fear responses. Muscimol-treated rats froze 
A

\begin{tabular}{|lccccccc|}
\hline Groups & Day 1 & Day 2 & Day 3 & Day 4 & Day 5 & Day6 & Day 7 \\
\hline No Context Extinction & 2 CS - US & CS & CTX - US & & CTX - US & CS & CS \\
\hline Context Extinction & 2 CS - US & CS & CTX - US & CTX & CTX - US & T CS & CS \\
\hline
\end{tabular}

BLA infusion of vehicle or muscimol ( $0.3 \mu \mathrm{g}$ in $0.3 \mu \mathrm{L}$, both sides).

B

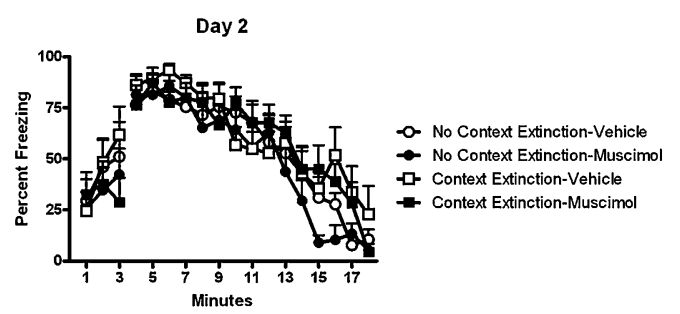

C

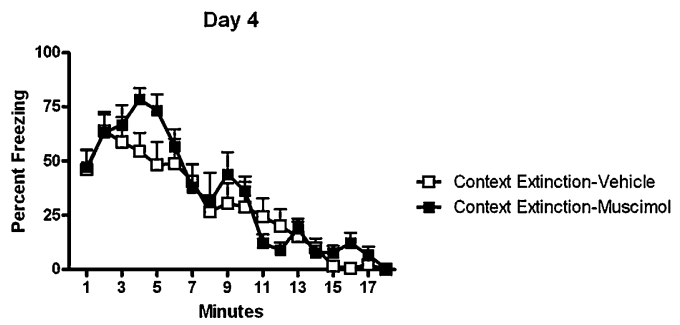

D

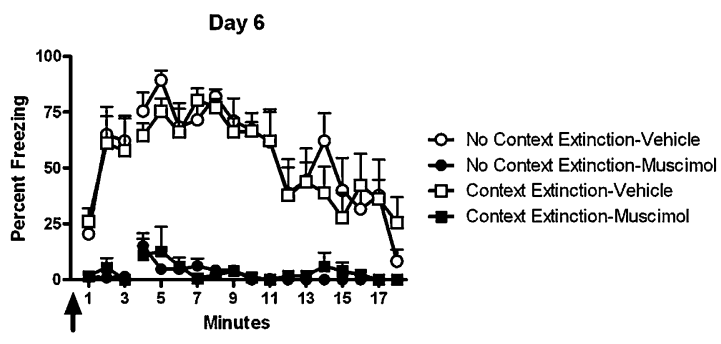

E

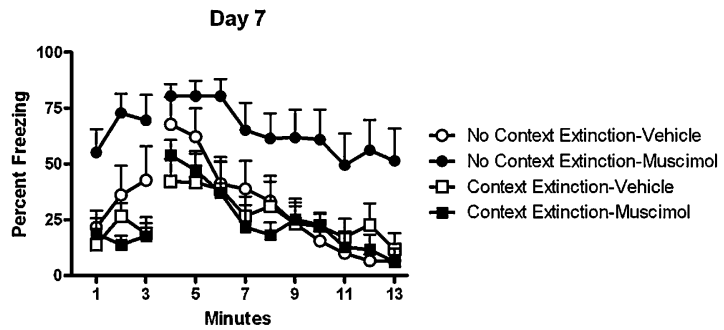

Figure 5. Inactivation of the BLA does not impair learning extinction of reinstated fear if the reinstatement context has been extinguished. $(A)$ Description of the behavioral procedures used in Experiment 4 (CS: flashing light conditioned stimulus; US: footshock unconditioned stimulus; CTX: context). (B) All illustrations show the mean and SEM levels of freezing. All rats lost freezing responses across initial extinction. (C) Rats lost freezing responses to the context in which US-alone exposure occurred (group Context Extinction). (D) Rats infused with vehicle before their second (groups No Context Extinction-Vehicle and Context Extinction-Vehicle) extinction lost freezing responses across the session. BLA infusion of muscimol reduces fear responses (groups No Context Extinction-Muscimol and Context Extinction-Muscimol). (E) The retention test revealed that BLA inactivation does not impair learning extinction of reinstated fear if the context in which US-alone exposure occurred had been extinguished once (group Context Extinction-Muscimol).

significantly less to the CS than vehicle-treated rats $\left(F_{(1,21)}=177.1\right.$, $P<0.05)$. There was no significant difference between rats in groups No Context Extinction-Muscimol and Context ExtinctionMuscimol $\left(F_{(1,21)}=0.0, P>0.05\right)$. The depression of fear responses induced by muscimol was also present before the onset of the CS. Muscimoltreated rats froze significantly less than vehicle-treated rats $\left(F_{(1,21)}=57.8, P<\right.$ $0.05)$. Overall, levels of freezing significantly and gradually declined across the re-extinction session $\left(F_{(1,21)}=44.9, P<\right.$ $0.05)$.

The retention test data on day 7 (Fig. 5E) demonstrate that initial extinction of the reinstatement context renders second extinction of the visual CS BLA independent. Rats in group Context Extinction-Muscimol showed similar levels of CS freezing than rats in group Context Extinction-Vehicle $\left(F_{(1,21)}=0.0, P>\right.$ 0.05). In contrast, re-extinction of the CS requires neuronal activity in the BLA if the reinstatement context has not been extinguished. Rats in group No Context Extinction-Muscimol froze significantly more to the CS than rats in group No Context Extinction-Vehicle $\left(F_{(1,21)}=10.7\right.$, $P<0.05)$. There was no difference between vehicle-treated rats across the CS retention test $\left(F_{(1,21)}=0.1, P>0.05\right)$. Rats treated with muscimol froze significantly more to the CS if they had not received the context-alone exposure on day 4 (groups No Context Extinction-Muscimol vs. Context Extinction-Muscimol; $\left.F_{(1,21)}=14.0, P<0.05\right)$. Levels of pre-CS freezing revealed that muscimol prevented the loss of fear to the reinstatement context when it had not been presented by itself on day 4 . Rats in group No Context Extinction-Muscimol froze significantly more than the remaining groups $\left(F_{(1,21)}=96.3, P<0.05\right)$. There was no difference between the remaining groups of rats $(F s<1.4)$.

\section{Discussion}

The present series of experiments studied the role of the BLA in the reinstatement of extinguished fear responses by USalone exposure. The experiments had two aims. The first was to show that the BLA is critical for the context conditioning by US-alone exposure, which mediates the reinstatement of fear responding when an extinguished CS is subsequently tested in the now dangerous context. The results of Experiment 1 confirmed this role of the BLA. Rats subjected to an intraBLA infusion of muscimol before US-alone exposure subsequently failed to show the reinstated fear responses present in control rats subsequently tested with the extinguished CS in the context where the US-alone exposure had occurred. Experiment 1 also replicated previous demonstrations that the BLA is critical for the restoration of fear responses produced by pairing an extinguished CS with the US (Laurent and Westbrook 2009b). Rats subjected to a BLA infusion of muscimol before that pairing 
subsequently failed to show the fear responses restored by pairing the extinguished CS with the US in control rats. These results confirm and extend the role of the BLA in the acquisition of fear responses: The BLA is critical for learning to fear a novel CS or context, a context rendered familiar by pre-exposure, as well as relearning to fear an extinguished CS or context (Lee and Kim 1998; Wilensky et al. 2006; Laurent and Westbrook 2009b). These results also show for the first time, as far as we are aware, that the BLA is also critical for the reinstatement of fear responses to an extinguished CS. This conclusion is consistent with the previously mentioned role of the BLA in conditioning or reconditioning of fear to a context by US-alone exposures. It is also consistent with the evidence documenting the dependence of reinstatement on testing the extinguished CS in a context rendered dangerous by the US-alone exposure (Bouton and Bolles 1979; Westbrook et al. 2002).

The second aim of the present experiments was to examine the role of the BLA in extinction of the fear responses reinstated by US-alone exposure. This question originates in recent findings that have shown that the BLA is critical for learning the inhibition produced by extinction, but not for relearning this inhibition (Kim and Richardson 2008; Laurent and Westbrook 2008; Laurent et al. 2008). Rats whose fear responses are extinguished under a BLA infusion of muscimol show fear responses when subsequently tested drug-free. However, rats whose fear responses have been restored by pairing an extinguished cue (discrete stimulus or context CS) with the US and whose fear responses are again extinguished under a BLA infusion of muscimol, subsequently show just as few fear responses as control rats re-extinguished or extinguished under vehicle. In Experiment 2, we replicated these findings. We did so in order to ensure their reliability in the procedures to be used in the remaining experiments. In Experiment 2, rats that received their first extinction session under BLA infusion of muscimol exhibited fewer fear responses across that session, but substantially more fear responses than control rats when tested the following day in the absence of the drug. These results confirm the critical role of the BLA in the expression of conditioned fear responses (Helmstetter and Bellgowan 1994; Muller et al. 1997; Lee and Kim 1998; Lee et al. 2001; Maren et al. 2001; Wilensky et al. 2006), and in learning the inhibition produced by extinction, which mediates long-term suppression of fear responses (Kim and Richardson 2008; Laurent and Westbrook 2008; Laurent et al. 2008). More importantly, Experiment 2 also confirmed that relearning this inhibition can occur in the absence of neuronal activity in the BLA. When infused in the BLA with muscimol before their second extinction that followed reconditioning, rats again froze less than controls but showed comparable low levels of freezing as controls when tested drug-free the following day.

In Experiments 3 and 4, we studied the role of the BLA in relearning inhibition when fear responses to an extinguished CS had been reinstated by US-alone exposure. In Experiment 3, we again confirmed that the BLA was critical for learning to inhibit fear responses across extinction. Rats were trained to fear a CS, subjected to US-alone exposure in the absence of prior CS extinction, and then administered their first extinction under a BLA infusion of muscimol. These rats showed less fear responses across that extinction, but substantially more fear responses on the subsequent retention test than control rats extinguished for the first time under vehicle. However, exactly the same outcome was observed in the rats that had received CS extinction before the US-alone exposure and that were then subjected to extinction of reinstated fear responses under a BLA infusion of muscimol. These rats also showed less fear responses across that re-extinction than vehicle-treated rats, confirming the role of the BLA in the expression of fear responses either conditioned, reconditioned (Laurent and Westbrook 2008; Laurent et al. 2008) or, in this case, reinstated by US-alone exposure. But more importantly, for present purposes, the muscimol infused rats not only showed substantially more fear responses on the subsequent retention test than rats re-extinguished under vehicle, but just as many fear responses as did rats infused with muscimol before their initial extinction. Thus, these results suggest that relearning to inhibit the fear responses reinstated by US-alone exposure is BLA dependent, whereas relearning to inhibit the fear responses restored by pairing of the extinguished CS with the US is BLA independent.

However, in Experiment 3, the fear responses reinstated to the extinguished CS by the US-alone exposure were extinguished in the context where the US alone had occurred. Hence, rats infused with muscimol into the BLA could have relearned to inhibit fear responses to the CS, since it had previously been extinguished. In contrast, they may have failed to learn to inhibit fear responses to the conditioned context. To be sure, the context had been extinguished previously, but only when it had been in the background across extinction of the CS, not when it had been the sole recipient of the fear conditioning. If the muscimol infused rats had failed to learn to inhibit fear responses to the conditioned context, then they would have continued to show fear responses when subsequently tested with the CS because they were tested in a context whose association with the US was intact. The association produced by the US-alone exposure would have been intact, since the BLA is critical for rats learning to inhibit fear responses across the initial extinction of context conditioned fear (Laurent and Westbrook 2008; Laurent et al. 2008). But the BLA is not necessary for relearning to inhibit fear responses across re-extinction of a conditioned context.

Therefore, in Experiment 4, we examined the role of the BLA in relearning to inhibit fear responses in rats with a differential history of context extinction. The design consisted of administering US-alone exposures in order to reinstate fear responses to an extinguished CS and then subjecting one group, but not a second group, to extinction of the context fear conditioned by the USalone exposure. US-alone exposure was again administered to both groups and the reinstated fear responses to the extinguished CS were re-extinguished under a BLA infusion of muscimol or vehicle. We replicated the results obtained in the previous experiment with rats that did not receive the context extinction between the two US-alone episodes. Those infused with muscimol before extinction of reinstated fear responses to the CS froze less across that session, but substantially more than the vehicle-treated rats when subsequently tested with the CS. Rats that had received the context extinction between the two US-alone episodes also showed few fear responses when subjected to extinction of the reinstated CS in comparison to the vehicle-treated rats. Importantly, both groups of rats showed similar low levels of freezing on the subsequent retention test. Thus, the prior extinction of the context fear produced by the US-alone exposure allowed the second extinction of the conditioned context to occur in the absence of neuronal activity in the BLA (Laurent and Westbrook 2008; Laurent et al. 2008).

Various lines of evidence have led to a neural model of fear conditioning, which holds that sensory information about the CS (either a discrete stimulus or the array of cues comprising a context) and the shock US converge on neurons in the BLA, which project to fear output neurons in the central nucleus of the amygdala (CeA) (Maren 2001). The concomitant activation of BLA neurons by CS and US inputs potentiates synaptic plasticity via NMDAr activation (Rodrigues et al. 2004). The fear output neurons in the $\mathrm{CeA}$, in turn, project to diencephalic and brain stem structures, which control the behavioral, autonomic, and endocrine components of the fear response (Sah et al. 2003). Consistent with this model, disruption of neuronal activity in the 
BLA before US-alone exposure impaired the association normally formed between the context and that US. This impairment was evidenced by low levels of freezing when the rats were returned to that context, as well as low levels of freezing when they were then tested with the extinguished CS for reinstatement produced by US-alone exposure.

Contemporary neural models hold that interactions between the BLA and the infralimbic (IL) region of the medial prefrontal cortex mediate acquisition of inhibition across extinction, its consolidation, and subsequent retrieval (Quirk and Mueller 2008). Specifically, the BLA is required for acquisition and consolidation of this inhibition (Lin et al. 2003; Herry et al. 2006; Kim et al. 2007; Sotres-Bayon et al. 2007, 2009; Laurent and Westbrook 2008; Laurent et al. 2008), whereas the IL is necessary for its consolidation and retrieval (Quirk et al. 2000; Milad and Quirk 2002; Santini et al. 2004; Hugues et al. 2006; Sierra-Mercado et al. 2006; Burgos-Robles et al. 2007; Laurent and Westbrook 2008, 2009a; Sotres-Bayon et al. 2009). This inhibition occurs through projection of IL neurons to intercalated cells located between the BLA and the CeA, thereby suppressing the output neurons in the CEA which co-ordinate the various components of the fear responses (Pare and Smith 1993; McDonald 1998; Royer et al. 1999). Consistent with this model, disruption of neuronal activity in the BLA impaired the long-term inhibition of fear responses produced by extinction of a dangerous context (Experiment 3 ) or dangerous CS (Experiment 2). However, relearning that inhibition across second extinction can occur independent of neuronal activity within the BLA (Experiments 2 and 4). Laurent and Westbrook (2009a) provided evidence that this relearning involves compensatory mechanisms in the IL. These investigators reported that IL inactivation disrupts acquisition, consolidation, and retrieval of the inhibition produced by second extinction. These findings are also consistent with the model just described. This model suggests that, once established by initial extinction, the inhibitory circuitry in the IL is activated and strengthened across second extinction when neuronal activity in the BLA is disrupted. This implies that the establishment of this circuitry by context extinction in Experiment 4 supported relearning to inhibit fear responses across the second extinction of that context. This relearning of inhibition undermined the ability of the context to reinstate extinguished fear responses to the extinguished CS at test.

\section{Materials and Methods}

\section{Subjects}

Subjects were 140 experimentally naïve male Wistar rats (rattus norvegicus). They were obtained from a local supplier (Animal Resource Center) and weighed between 250 and $350 \mathrm{~g}$. They were housed in plastic boxes $(22 \mathrm{~cm}$ high $\times 65 \mathrm{~cm}$ long $\times 40 \mathrm{~cm}$ wide $)$ located in a climate-controlled colony room under natural lighting. There were eight rats per box with food and water continuously available. Three days after arrival in the laboratory, rats were handled each day for $4 \mathrm{~d}$. The experimental procedures were approved by the Animal Ethics Committee at the University of New South Wales and conducted in accordance with the National Institutes of Health Guidelines for the Care and Use of Laboratory Animals (publication DHHS NIH 86-23). All experiments occurred between 7:00 a.m. and 10:00 p.m.

\section{Drugs}

The $\mathrm{GABA}_{\mathrm{A}}$ receptor agonist muscimol (Sigma) was dissolved in $0.9 \%$ (wt/vol) nonpyrogenic saline to obtain a final concentration of $1 \mu \mathrm{g} / \mu \mathrm{L}$. Nonpyrogenic saline was used to control for any effect of the infusion procedures per se. The dose of muscimol was selected on the basis of previous work conducted in our laboratory (Laurent and Westbrook 2008; Laurent et al. 2008).

\section{Surgery and microinjections}

Rats received intraperitoneal (i.p.) injections of $1.3 \mathrm{~mL} / \mathrm{kg}$ of the anesthetic ketamine (Ketapex; Apex Laboratories) at a concentration of $100 \mathrm{mg} / \mathrm{mL}$ and of $0.3 \mathrm{~mL} / \mathrm{kg}$ of the muscle relaxant xylazine (Rompun; Bayer) at a concentration of $20 \mathrm{mg} / \mathrm{mL}$. Anaesthetized rats were then mounted on a stereotaxic apparatus (Kopf Instruments), and 26 gauge guide cannulas (Plastics One) were implanted through holes drilled in both hemispheres of the skull. The tips of the guide cannulas were aimed bilaterally at the basolateral amygdala using the following coordinates: $2.3 \mathrm{~mm}$ posterior to bregma, $4.8-5 \mathrm{~mm}$ lateral to the midline, and 7.7$8 \mathrm{~mm}$ ventral to the skull. The guide cannulas were maintained in position with dental cement and dummy cannulas were kept in each guide at all times except during microinjections. Immediately after the surgical procedure, rats were injected i.p. with a prophylactic $(0.3 \mathrm{~mL})$ dose of $300 \mathrm{mg} / \mathrm{kg}$ solution of procaine penicillin. Rats were allowed $4 \mathrm{~d}$ to recover from surgery, during which time they were handled and weighed daily.

Muscimol $(1 \mu \mathrm{g} / \mu \mathrm{L})$ and vehicle were infused bilaterally in the basolateral amygdala by inserting a 33-gauge double-internal cannula into the double-guide cannula. The double-internal cannula was connected to a $25 \mu \mathrm{L}$ glass syringe attached to an infusion pump (Harvard Apparatus) and projected an additional $1 \mathrm{~mm}$ ventral to the tip of the guide cannula. A total volume of $0.3 \mu \mathrm{L}$ (each side) was delivered at a rate of $0.1 \mu \mathrm{L} / \mathrm{min}$. The double-internal cannula remained in place for an additional $1 \mathrm{~min}$ after the infusions and was then removed. On the day before infusions, the dummy cannula was removed and the infusion pump was turned on for $3 \mathrm{~min}$ in order to familiarize the rats with the procedure and thereby minimize any stress produced by this procedure when infusions occurred. All infusions occurred in a separate room from the colony room and the experimental room.

\section{Apparatus}

Four chambers, each measuring $26 \mathrm{~cm}$ height $\times 30 \mathrm{~cm}$ length $\times$ $30 \mathrm{~cm}$ width, were used for fear conditioning, extinction training, reinstatement, and testing. The walls of these chambers, as well as the hinged lid, were constructed of Perspex. The floor in each chamber consisted of stainless steel rods, $2 \mathrm{~mm}$ in diameter, spaced $10 \mathrm{~mm}$ apart (center to center). Each chamber was enclosed in a sound- and light-attenuated shell. The inside walls of the shells were painted black. A fan was mounted on the side wall of each shell to recycle air. A white fluorescent tube mounted at the back of each shell was used for the presentation of a 30-s light conditioned stimulus (CS, $\sim 57$ lux measured at the center of the chamber) flashing at a rate of $2.5 / \mathrm{s}$ during the otherwise dark session. The unconditioned stimulus (US) was a $1-\mathrm{s}, 0.8-\mathrm{mA}$ unscrambled AC $50-\mathrm{Hz}$ footshock from a constant-current generator that was delivered to the floor of each chamber. The current available to each floor could be adjusted using an in-line milliampere meter. The floor of each chamber was $3 \mathrm{~cm}$ above a tray of paper pellet bedding (Fibrecycle) that was changed between rats. After removal of a rat, the floor of each chamber was cleaned with a solution of acetic acid (1\%) to eliminate any residue and provide a distinctive odor. A camera mounted on the back wall of each shell was used to record the behavior of each rat. Each camera was connected to a monitor and a DVD recorder located in another room of the laboratory. This room contained the computer that controlled stimulus presentations via appropriate software (LabView, National Instruments).

\section{Behavioral procedures}

\section{Experiment 1: Inactivation of the BLA impairs reconditioning and reinstatement of extinguished fear}

On the morning of day 1 , rats were placed in conditioning chambers and received $3 \mathrm{~min}$ later a 30 -s presentation of the flashing light CS. This stimulus terminated in a $0.8-\mathrm{mA}, 1-\mathrm{s}$ footshock. Rats remained in the chambers for an additional 2 min before being returned to their home-cage. This procedure 
was repeated in the afternoon of day 1 . On day 2 , all rats received an extinction session that consisted of a 3-min adaptation period in the chambers, followed by a 15 -min continuous presentation of the CS in the absence of shock. On day 3, rats in groups Reconditioning-Muscimol and Reinstatement-Muscimol were infused with muscimol into the BLA $(0.3 \mu \mathrm{g} / 0.3 \mu \mathrm{L}$, each side), whereas those in groups Reconditioning-Vehicle and Reinstatement-Vehicle were infused with saline. Forty-five minutes later, rats in groups Reconditioning received a single conditioning session identical to that described on day 1 . In contrast, rats in groups Reinstatement were returned to the chambers and received an unsignaled footshock after $5 \mathrm{~min}$. Six hour later, rats that had been infused with muscimol now received an infusion of saline (groups ReconditioningMuscimol and Reinstatement-Muscimol), and rats that had been infused with saline now received an infusion of muscimol (groups Reconditioning-Vehicle and Reinstatement-Vehicle). These counterbalanced infusions were used to control for any effects of muscimol per se on responding across the subsequent test. On day 4 , all rats were tested for fear responses to the CS. Rats were placed in the chambers for $3 \mathrm{~min}$ followed by a 10-min presentation of the light CS in the absence of the US.

\section{Experiment 2: Inactivation of the BLA impairs extinction of conditioned fear but spares extinction of reconditioned fear}

On the morning of day 1 , rats were placed in conditioning chambers and received $30 \mathrm{~s}$ later a 30 -s presentation of the flashing light CS. This stimulus terminated in a 0.8-mA, 1-s footshock. Rats remained in the chambers for an additional 2 min before being returned to their home-cage. This procedure was repeated in the afternoon of day 1 . On day 2 , rats in groups Re-extinction received an extinction session that was the same as that described previously. In contrast, rats in groups Extinction did not receive extinction but were exposed to the chambers for $18 \mathrm{~min}$. On day 3 , all rats receive a single conditioning episode identical to that on day 1. On day 4, rats in groups Extinction-Muscimol and Re-extinction-Muscimol were infused with muscimol into the BLA $(0.3 \mu \mathrm{g} / 0.3 \mu \mathrm{L}$, each side), whereas those in groups Extinction-Vehicle and Re-extinction-Vehicle were infused with saline. Forty-five minutes after infusion, all rats received an extinction session identical to that described on day 2 for groups Reextinction. Counterbalanced infusions were given $6 \mathrm{~h}$ later in the manner described. On day 5 , all rats were tested in the manner described.

\section{Experiment 3: Inactivation of the BLA impairs learning extinction of reinstated fear}

On day 1, all rats received two conditioning episodes in the manner described in Experiment 2. On day 2, rats in groups Control Re-extinction and Re-extinction received an extinction session that was the same as that described. Rats in groups Extinction did not receive extinction but were exposed to the chambers for $18 \mathrm{~min}$. On day 3, all rats were returned to the chambers and received an unsignaled footshock after $5 \mathrm{~min}$. They remained in the chambers for an additional $5 \mathrm{~min}$ before being returned to their home-cage. On day 4, rats in groups ExtinctionMuscimol and Re-extinction-Muscimol received an infusion of muscimol $(0.3 \mu \mathrm{g} / 0.3 \mu \mathrm{L}$, each side) in the BLA, whereas those in groups Extinction-Vehicle and Re-extinction-Vehicle received saline. Half of the rats in group Control Re-extinction received an infusion of muscimol, whereas the other half received saline. Forty-five minutes after infusion, rats in groups Extinction and Reextinction received an extinction session identical to that on day 2. Rats in group Control Re-extinction did not receive extinction but were handled. Counterbalanced infusions were given $6 \mathrm{~h}$ later in the manner described. On day 5 , all rats were tested in the manner described.

\section{Experiment 4: Inactivation of the BLA does not impair learning extinction} of reinstated fear if the reinstatement context has been extinguished

Rats received two conditioning episodes on day 1 and an extinction session on day 2 in the manner described in Experiment 1. On day 3, rats received an unsignaled exposure to the footshock identical to that previously described. On day 4, rats in groups Context Extinction were exposed to the chambers for $18 \mathrm{~min}$ in the absence of shock. Rats in group No Context Extinction were not exposed to the chambers but were handled. On day 5 , all rats received an unsignaled exposure to the footshock identical to that on day 3. On day 6, rats in groups No Context Extinction-Muscimol and Context Extinction-Muscimol received an infusion of muscimol $(0.3 \mu \mathrm{g} / 0.3 \mu \mathrm{L}$, each side $)$ in the BLA, whereas those in groups No Context Extinction-Vehicle and Context ExtinctionVehicle received saline. Forty-five minutes after infusion, all rats received an extinction session identical to that on day 2 . Counterbalanced infusions were given $6 \mathrm{~h}$ later in the manner described. On day 7 , all rats were tested in the manner described.

\section{Histology}

Subsequent to behavioral testing, subjects implanted with cannulas received a lethal dose of sodium pentobarbital and their brains were removed and frozen. Unfixed brains were sectioned coronally at $40 \mu \mathrm{m}$ through the basolateral amygdala. Every third section was collected on a slide, and the sections were stained with cresyl violet. The approximate location of injection cannulas tips was determined under a microscope by a trained observer who was unaware of the subjects' group designations using the boundaries defined by the Paxinos and Watson atlas (Paxinos and Watson 2007). Subjects with inaccurate cannula placements or with extensive damage to the basolateral amygdala were excluded from the statistical analysis. Importantly, the behavior exhibited by these subjects did not follow any particular trend. Thus, the data are not discussed here.

\section{Scoring and statistics}

Freezing was defined as the absence of all movements, except those related to breathing (Blanchard and Blanchard 1969). The behavior of each rat was recorded on videotape, and freezing was rated with a time-sampling procedure in which each rat was observed every $2 \mathrm{~s}$ and scored as either freezing or moving. A percentage score was calculated for the proportion of the total observation that each rat spent freezing. Freezing was rated by two observers, one of whom was unaware of the subject's group designation. There was a high degree of agreement between the two observers: The Pearson product moment correlation between their rating was $>0.95$. The data were analyzed in each experiment by a set of planned nonorthogonal contrasts that controlled the error rate using the Bonferroni inequality procedure. Significance was set at the 0.05 level. The behavior of each experimental group was represented as percent levels of freezing per minute.

\section{Acknowledgments}

This research was supported by an Australian Research Council grant (DP0881385). We thank Genevra Hart for her helpful discussions.

\section{References}

Blanchard RJ, Blanchard DC. 1969. Crouching as an index of fear. J Comp Physiol Psychol 67: 370-375.

Bouton ME, Bolles RC. 1979. Role of conditioned contextual stimuli in reinstatement of extinguished fear. J Exp Psychol Anim Behav Process 5: 368-378.

Bouton ME, Rosengard C, Achenbach GG, Peck CA, Brooks DC. 1993. Effects of contextual conditioning and unconditional stimulus presentation on performance in appetitive conditioning. QJ Exp Psychol B 46: 63-95.

Bouton ME, Westbrook RF, Corcoran KA, Maren S. 2006. Contextual and temporal modulation of extinction: Behavioral and biological mechanisms. Biol Psychiatry 60: 352-360.

Burgos-Robles A, Vidal-Gonzalez I, Santini E, Quirk GJ. 2007. Consolidation of fear extinction requires NMDA receptor-dependent bursting in the ventromedial prefrontal cortex. Neuron 53: 871-880.

Harris JA, Jones ML, Bailey GK, Westbrook RF. 2000. Contextual control over conditioned responding in an extinction paradigm. J Exp Psychol Anim Behav Process 26: 174-185. 
Helmstetter FJ, Bellgowan PS. 1994. Effects of muscimol applied to the basolateral amygdala on acquisition and expression of contextual fear conditioning in rats. Behav Neurosci 108: 1005-1009.

Herry C, Trifilieff P, Micheau J, Luthi A, Mons N. 2006. Extinction of auditory fear conditioning requires MAPK/ERK activation in the basolateral amygdala. Eur J Neurosci 24: 261-269.

Hugues S, Chessel A, Lena I, Marsault R, Garcia R. 2006. Prefrontal infusion of PD098059 immediately after fear extinction training blocks extinction-associated prefrontal synaptic plasticity and decreases prefrontal ERK2 phosphorylation. Synapse 60: 280-287.

Kim JH, Richardson R. 2008. The effect of temporary amygdala inactivation on extinction and reextinction of fear in the developing rat: Unlearning as a potential mechanism for extinction early in development. J NeurosC 28: 1282-1290.

Kim J, Lee S, Park H, Song B, Hong I, Geum D, Shin K, Choi S. 2007. Blockade of amygdala metabotropic glutamate receptor subtype 1 impairs fear extinction. Biochem Biophys Res Commun 355: 188-193.

Laurent V, Westbrook RF. 2008. Distinct contributions of the basolateral amygdala and the medial prefrontal cortex to learning and relearning extinction of context conditioned fear. Learn Mem 15: 657-666.

Laurent V, Westbrook RF. 2009a. Inactivation of the infralimbic but not the prelimbic cortex impairs consolidation and retrieval of fear extinction. Learn Mem 16: 520-529.

Laurent V, Westbrook RF. 2009b. Infusion of the NMDA receptor antagonist, DL-APV, into the basolateral amygdala disrupts learning to fear a novel and a familiar context as well as relearning to fear an extinguished context. Learn Mem 16: 96-105.

Laurent V, Marchand AR, Westbrook RF. 2008. The basolateral amygdala is necessary for learning but not relearning extinction of context conditioned fear. Learn Mem 15: 304-314.

LeDoux J. 2003. The emotional brain, fear, and the amygdala. Cell Mol Neurobiol 23: 727-738.

Lee H, Kim JJ. 1998. Amygdalar NMDA receptors are critical for new fear learning in previously fear-conditioned rats. J Neurosci 18: 8444-8454

Lee HJ, Choi JS, Brown TH, Kim JJ. 2001. Amygdalar nmda receptors are critical for the expression of multiple conditioned fear responses. $J$ Neurosci 21: 4116-4124.

Leung HT, Westbrook RF. 2008. Spontaneous recovery of extinguished fear responses deepens their extinction: A role for error-correction mechanisms. J Exp Psychol Anim Behav Process 34: 461-474.

Lin CH, Yeh SH, Lu HY, Gean PW. 2003. The similarities and diversities of signal pathways leading to consolidation of conditioning and consolidation of extinction of fear memory. J Neurosci 23: 8310-8317.

Maren S. 2001. Neurobiology of Pavlovian fear conditioning. Annu Rev Neurosci 24: 897-931.

Maren S, Yap SA, Goosens KA. 2001. The amygdala is essential for the development of neuronal plasticity in the medial geniculate nucleus during auditory fear conditioning in rats. J Neurosci 21: RC135-1-6.

McDonald AJ. 1998. Cortical pathways to the mammalian amygdala. Prog Neurobiol 55: 257-332.
Milad MR, Quirk GJ. 2002. Neurons in medial prefrontal cortex signal memory for fear extinction. Nature 420: 70-74.

Muller J, Corodimas KP, Fridel Z, LeDoux JE. 1997. Functional inactivation of the lateral and basal nuclei of the amygdala by muscimol infusion prevents fear conditioning to an explicit conditioned stimulus and to contextual stimuli. Behav Neurosci 111: 683-691.

Myers KM, Davis M. 2007. Mechanisms of fear extinction. Mol Psychiatry 12: $120-150$

Pare D, Smith Y. 1993. The intercalated cell masses project to the central and medial nuclei of the amygdala in cats. Neuroscience 57: 10771090.

Paxinos G, Watson C. 2007. The rat brain in stereotaxic coordinates. Academic, Sydney, Australia.

Quirk GJ, Mueller D. 2008. Neural mechanisms of extinction learning and retrieval. Neuropsychopharmacology 33: 56-72.

Quirk GJ, Russo GK, Barron JL, Lebron K. 2000. The role of ventromedial prefrontal cortex in the recovery of extinguished fear. J Neurosci 20: 6225-6231.

Rodrigues SM, Schafe GE, LeDoux JE. 2004. Molecular mechanisms underlying emotional learning and memory in the lateral amygdala. Neuron 44: 75-91.

Royer S, Martina M, Pare D. 1999. An inhibitory interface gates impulse traffic between the input and output stations of the amygdala. J Neurosci 19: $10575-10583$.

Sah P, Faber ES, Lopez De Armentia M, Power J. 2003. The amygdaloid complex: Anatomy and physiology. Physiol Rev 83: 803-834.

Santini E, Ge H, Ren K, Pena de Ortiz S, Quirk GJ. 2004. Consolidation of fear extinction requires protein synthesis in the medial prefrontal cortex. J Neurosci 24: 5704-5710.

Sierra-Mercado Jr., D, Corcoran KA, Lebron-Milad K, Quirk GJ. 2006. Inactivation of the ventromedial prefrontal cortex reduces expression of conditioned fear and impairs subsequent recall of extinction. Eur J Neurosci 24: 1751-1758.

Sotres-Bayon F, Bush DE, LeDoux JE. 2007. Acquisition of fear extinction requires activation of NR2B-containing NMDA receptors in the lateral amygdala. Neuropsychopharmacology 32: 1929-1940.

Sotres-Bayon F, Diaz-Mataix L, Bush DE, LeDoux JE. 2009. Dissociable roles for the ventromedial prefrontal cortex and amygdala in fear extinction: NR2B contribution. Cereb Cortex 19: 474-482.

Westbrook RF, Iordanova M, McNally G, Richardson R, Harris JA. 2002. Reinstatement of fear to an extinguished conditioned stimulus: Two roles for context. J Exp Psychol Anim Behav Process 28: 97110.

Wilensky AE, Schafe GE, Kristensen MP, LeDoux JE. 2006. Rethinking the fear circuit: The central nucleus of the amygdala is required for the acquisition, consolidation, and expression of Pavlovian fear conditioning. J Neurosci 26: 12387-12396.

Received October 5, 2009; accepted in revised form November 21, 2009. 


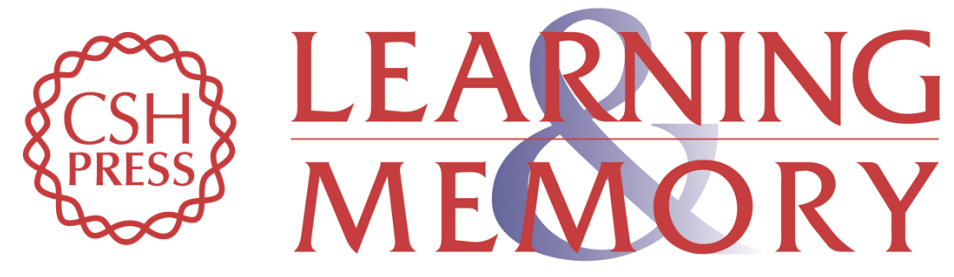

\section{Role of the basolateral amygdala in the reinstatement and extinction of fear responses to a previously extinguished conditioned stimulus}

Vincent Laurent and R. Frederick Westbrook

Learn. Mem. 2010, 17:

Access the most recent version at doi:10.1101/lm.1655010

References This article cites 38 articles, 12 of which can be accessed free at:

http://learnmem.cshlp.org/content/17/2/86.full.html\#ref-list-1

License

Email Alerting Receive free email alerts when new articles cite this article - sign up in the box at the Service top right corner of the article or click here. 